\title{
A Novel Retrospective Gating Method for Intracoronary Ultrasound Images based on Image Properties
}

\author{
SA de Winter, R Hamers, M Degertekin, K Tanabe, PA Lemos, PW Serruys, JRTC Roelandt, \\ N Bruining
}

Erasmus Medical Centre, Rotterdam, The Netherlands

\begin{abstract}
Intracoronary ultrasound (ICUS) provides highresolution tomographic images of selected segments of coronary arteries. Series of cross-sectional images are acquired with motorized pullback imaging catheters and used for quantitative analysis in intracoronary ultrasound studies (ICUS). Due to catheter displacement in the vascular lumen during the cardiac cycle the images that are typically acquired at $0.5 \mathrm{~mm} / \mathrm{s}$ are anatomically shuffled. This results in a saw-tooth shaped appearance of the coronary segment in longitudinal reconstructed views (L-views) used frequently in quantitative coronary ultrasound (QCU) software. This paper describes a novel image-based gating method called "Intelligate", which overcomes this problem by automatic retrospective selection of end-diastolic frames from pre-recorded ICUS studies. Our evaluation shows that there are no quantitative differences between analysis results of hardware ECG-gated and intelligated ICUS studies.
\end{abstract}

\section{Introduction}

Intracoronary ultrasound (ICUS) gives insight into the composition and extent of atherosclerotic plaque[1]. Previous studies have shown that ICUS may visualize atherosclerotic plaques in angiographically normal coronary arteries. ICUS is used in clinical trials to evaluate the results of novel catheter-based interventional techniques as well as pharmaceutical treatments.

Cross-sectional ICUS images are acquired during a continuous speed pullback of the catheter in the coronary artery. In quantitative analysis procedures vessel wall borders are traced in a consecutive number of images. To assess vessel wall morphology and to facilitate quantitative analysis, three-dimensional (3D) reconstruction is performed using quantitative coronary ultrasound (QCU) software that visualizes segments by longitudinal cut-planes (L-views). This procedure avoids the time-consuming manual tracing of a series of individual cross-sections. However, cyclic systolicdiastolic changes of vascular dimensions and catheter motion result in saw-tooth shaped image artifacts in the
L-views that significantly hamper the quantitative analysis. (Semi-)automatic contour detection is interfered, the analysis process becomes time-consuming and the procedure may produce inaccurate results.

Most QCU analysis software packages acquire ICUS images stored on videotape at a rate of 1 frame per two seconds, randomly within the cardiac cycle, resulting in 1 $\mathrm{mm}$ intervals between the frames (assuming the catheter is pulled back with $0.5 \mathrm{~mm} / \mathrm{s}$ ), for use in area measurement and subsequent calculation of volumetric quantitative parameters. However, it has been reported that longitudinal catheter motion of more than $5 \mathrm{~mm}$ may occur during the cardiac cycle.

Previous studies have shown that an on-line ECGgated pullback procedure overcomes this motion problem and allows more accurate and reproducible measurements[2]. However, the technology requires expensive hardware, long setup times and considerably prolongs the acquisition procedure. Most laboratories still use non-gated acquisition and most existing image databases lack ECG-gated-data. Therefore, we developed the fully automated retrospective image-based gating method "Intelligate", that can select end-diastolic ICUS frames enabling fast and accurate analysis of ICUS studies. In this paper we describe this new method and its validation.

\section{The Intellgate method}

The Intelligate method selects ICUS images recorded in the end-diastolic phase. The rationale for selecting this phase is the mutual comparability of the images as the heart is relatively motionless here and blood flow has ceased. This means that forces originating from cardiac motion and the blood flow are no longer acting on the catheter. In the end-diastolic phase an imaging catheter that is not pulled back always resumes the same position in the lumen. During other phases in the cardiac cycle, the catheter position in the coronary artery may be different, which makes it difficult to determine the absolute distances between the consecutive ICUS frames in a non-ECG-gated study. Nevertheless, currently all QCU software packages neglect the catheter motion 
induced by the cardiac motion.

The Intelligate method is based on identification of the near end-diastolic images from information that is present in the images, and analyzes the changes of this information over time. The method does not need a simultaneously recorded ECG- or any other physiological reference trace.

\subsection{The Intelligate algorithm}

The Intelligate algorithm can be subdivided into three main steps: Pre-processing, Frame Classification and Final Selection. The processes can be interpreted as solving a classification problem having two disjunct classes with a discriminating property of association with the end-diastolic phase or not.

Pre-processing mostly focuses on extracting information from the image series that is used to construct feature vectors describing individual frames and sequences of frames. For some features filtering operations such as binarization based on edge gradients, are performed to enhance important image characteristics before calculation. One of the most prominent metrics is the similarity between images, in Intelligate expressed by the normalized 2D cross-correlation having the form:

$$
r=\frac{\sum_{i=1}^{M} \sum_{j=1}^{N}\left(a_{i j}-\bar{a}\right)\left(b_{i j}-\bar{b}\right)}{\sqrt{\sum_{i=1}^{M} \sum_{j=1}^{N}\left(a_{i j}-\bar{a}\right)^{2} \sum_{i=1}^{M} \sum_{j=1}^{N}\left(b_{i j}-\bar{b}\right)^{2}}}
$$

for $M \mathrm{x} N$ sized images $\mathrm{A}$ and $\mathrm{B}$ having pixels $a_{i j}$ and $b_{i j}$ on an 8-bit greyscale and $\bar{a}$ and $\bar{b}$ as the mean grey level. Analogous, many other image metrics are calculated and both time-domain and frequency domain properties after application of the fast Fourier transformation (FFT) to the feature signal are used to construct the feature vector. All attributes are normalized to remove biases associated with different scales using the simple equation:

$$
f_{i}^{\text {norm }}=\frac{f_{i}-\mu_{i}}{\sigma_{i}}
$$

with $f_{i}$ the $i$-th feature and $\mu_{i}$ and $\sigma_{i}$ the sampling mean and sampling variance respectively. From all features a best classifying feature vector is selected to reduce the number of attributes and hence the dimensionality of the problem.

Frame Classification handles the non-trivial task of judging whether the given feature vector is candidate for the end-diastolic class. Since Intelligate does not have to be trained before usage as in traditional machine learning, classification cannot be done by pattern matching using comparison to known vector patterns. Instead, relational properties and the degree of agreement between feature attributes are used in a statistical framework to quantify the confidence for a possible selection of an end-diastolic frame. This decision is strongly dependent on the a priori knowledge of general motion dynamics of the human heart and its related periodic behaviour. Classification is carried out with Nearest neighbour search based on a weighted Euclidean distance metric:

$$
d\left(\vec{v}_{1}, \vec{v}_{2}\right)=\sqrt{\sum_{i=1}^{k} \omega_{i}\left(v_{1, i}-v_{2, i}\right)^{2}}
$$

for $k$ features with $v_{1}$ and $v_{2}$ feature vectors and $\omega_{1}$ the weight factor.

The Final Selection step searches the time-domain of the candidate feature vectors in boxed intervals determined by the periodic properties of the heart cycle to select the actual end-diastolic frames from the candidate set.

\subsubsection{Internal validation process}

To validate the internal consistency of the method for each individually processed ICUS study, the mean heartbeat frequencies before (pre-Intelligate) and after gating are calculated for comparison. The rationale for this is the fact that the heart rate of a patient as embedded in the image sequence is an independent property and may not change after application of Intelligate. The preIntelligate phase examines the values of different features as found in the individual ICUS images with spectrum analysis techniques. This allows for the identification of repetition frequencies of the appearance of these image features. Peaks in the power spectrum of the feature indicate large contributions from those periodic components corresponding to the repetition frequency of the original feature. The average period corresponds to the mean heart rate. The left image of figure 1 shows that the mean heart rate is approximately $73 \mathrm{bpm}$.

The Intelligate method selects the end-diastolic frames from the non-gated ICUS study and therefore the mean heart rate can be determined from the time distances between the selected frames as well (the right image in figure 1). If this post-Intelligate value resembles the preIntelligate heart rate, the image selection is valid in the sense that it is able to preserve the patient's heart rate. Conversely, inconsistent selection of frames inevitably leads to different frame time distances and the mean heart rate will diverge from the pre-Intelligate heart rate. Observation of the differences between both values gives an indication of the accuracy of the Intelligate process. If a difference is found, the ICUS study is not further processed and the observer is notified. 

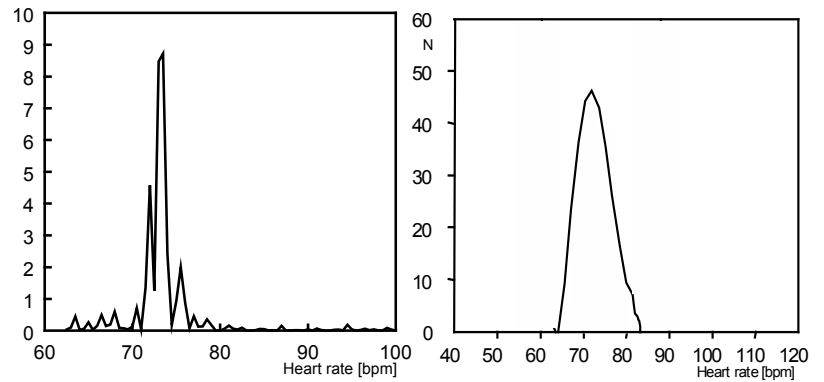

Figure 1. The left image shows a typical power spectrum derived from the ICUS study before gating. In this example, a strong peak is found at $73 \mathrm{bpm}$. On the right the distribution of the Intelligated frame rates converted to heartbeat are shown.

\subsubsection{Intelligate implementation}

Intelligate was initially executed unattended on a dedicated SGI Octane R12000 workstation (Silicon Graphics Inc., Mountain View, CA, USA) with 1.2 GB memory running the 64-bit IRIX operating system. The method is implemented in software and operates fully automatically. Currently, the software has been ported to an array of more commonly available Intel Pentium ${ }^{\mathrm{TM}}$ IV based computer systems in a parallel multi-pc architecture, which can handle large volumes of ICUS studies by the parallel processing principle. Average gating speed is now less than 5,5 minutes per 1000 frames for a single processing unit.

\subsection{Validation study}

\subsubsection{Data acquisition}

Fifteen randomly selected patients from the ITALICS trial - 6 months after implantation of a self-expanding coronary Wallstent (Schneider AB, Bülach, Switzerland) - were studied with ICUS. The ICUS studies were performed using mechanical sheath-based Boston Scientific Corporation ICUS catheters (BSC, Santa Clara, CA, USA) incorporating a $30 \mathrm{MHz}$ beveled singleelement transducer rotating at $1800 \mathrm{rpm}$. The sheath prevents direct contact of the imaging core with the vessel wall and increases catheter stability within the coronary artery. The ICUS studies were acquired in two passes: 1) the catheter was withdrawn at a continuous speed of $0.5 \mathrm{~mm} / \mathrm{s}$ using a motorized pullback system, 2) after this, the catheter was advanced to the distal position again and an ECG-gated pullback was performed with $0.2 \mathrm{~mm}$ discrete intervals. The non-gated studies were stored on S-VHS videotape at a rate of 25 images/s and the ECG-gated studies digitally on Magneto-Optical disks.

\subsubsection{ICUS analysis}

All ICUS studies, hardware ECG-gated and non-gated after application of Intelligate (see figure 2, panel B and
C), were analyzed with the CURAD (Curad BV, Wijk bij Duurstede, Netherlands) QCU analysis software[3]. The CURAD software focuses on detecting contours in Lviews. In most cases 4 L-views (containing 2 contours per view) are used to complete the analysis, in case of extreme vessel eccentricity a 5th L-view (containing a 9th contour) is added. The number of L-view contours is independent of the number of included individual crosssections in the ICUS study. Consequently, all available cross-sectional images are used in the analysis since they all contribute to the construction of the L-view. The contours traced correspond to lumen, stent and total vessel structures. Parameters for comparison between methods were lumen, stent, total vessel volumes, stent lengths and analysis times.

\subsubsection{Statistical analysis}

Quantitative data are presented as mean \pm standard deviation. Association between continuous variables was assessed by the Pearson correlation coefficient. Comparison of continuous variables was performed by the Student's two-tailed paired t-test. A p-value $<0.05$ was considered statistically significant. Relationship between two variables was analyzed by linear regression where applicable. Agreement of two methods was expressed as the mean difference \pm standard deviation between the two methods as proposed by Bland and Altman. For the internal validation of the Intelligate method the relationship between the found pre-processing and post-processing heart rates is analyzed with linear regression and Bland and Altman analysis.

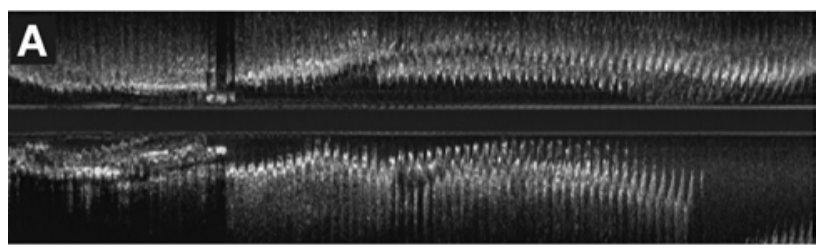

B

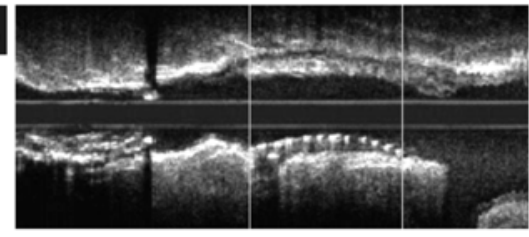

C

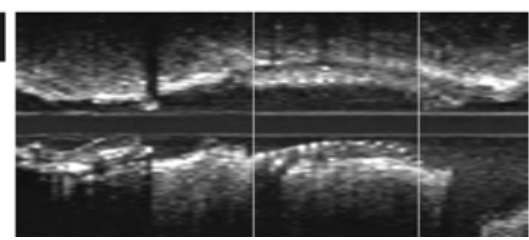

Figure 2. In panel A, a reconstructed longitudinal view (L-view) of a non-gated intracoronary ultrasound (ICUS) study is presented. Panel B shows an ICUS study of the same coronary segment but now acquired hardware ECG-gated. Finally, in panel C a L-view of the Intelligated data set is shown, that was extracted from images in the study shown in panel A. 


\section{Results}

Stent-, lumen- and vessel volumes were not significantly different between methods (ECG-gated vs. Intelligated) and were $225 \pm 86$ vs. $231 \pm 90,159 \pm 69$ vs. $160 \pm 74$ and $515 \pm 248$ vs. $495 \pm 245 \mathrm{~mm}^{3}$ respectively. The difference in measured stent lengths was $28 \pm 9$ vs. $27 \pm 8$ $\mathrm{mm}$, which was not statistically significant. There was no systematic difference for larger stents or their measured volumes. Relative differences were all within the 3,5\% deviation. The Bland-Altman analysis is presented in figure 3. The required time to perform the QCU analysis was $17 \pm 4$ minutes for hardware ECG-gated sets and $17 \pm 4$ minutes for sets processed with Intelligate.

The internal validation of the Intelligate method for each individual processed ICUS study showed a correlation of the mean heartbeat frequencies of 0.997 . Agreement between the values is expressed in a BlandAltman analysis. Differences were calculated relative to the mean value of the two obtained values for the R-R interval time. Variability, expressed as percentage of difference between methods, was $0 \pm 1.2 \%$. Mean absolute difference was $-4 \pm 10 \mathrm{~ms}$ with a standard error of the differences of $3 \mathrm{~ms}$.
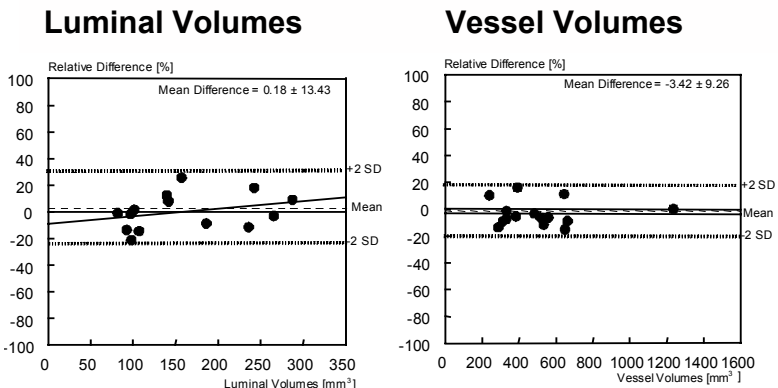

Figure 3. Bland and Altman plots for the comparison between hardware ECG-gated and Intelligated analysis results for lumen and vessel volumes.

\section{Discussion}

The described Intelligate method can be applied retrospectively to existing non-ECG gated ICUS studies and no additional equipment is needed in the catheterization laboratory. The current study shows that there are no statistically significant differences between the hardware ECG-gated method and the Intelligate method. One outlier in the stent length differences and one in the total vessel volume differences correspond to the same patient. In this patient, two stents have been implanted directly behind each other and the most distal stent was located in a strongly curved vessel causing difficulties in the stent length and in stent border detection.

This study also shows that QCU analysis after application of Intelligate is performed within a reasonable time as a result of the smooth appearance of the vessel wall morphology, which results in a much better performance of the applied automated contour detection algorithms. Observer interaction necessary to correct falsely detected contours, which is usually the case in non-gated studies, is significantly reduced, saving time and reducing intra- and inter-observer variability as a consequence.

The hardware image acquisition and selection process differs in important ways from that in Intelligate. Hardware ECG-gating acquires images at $0.2 \mathrm{~mm}$ discrete intervals, while continuous speed pullbacks are performed at $0.5 \mathrm{~mm} / \mathrm{s}$. The average heart rate of most patients is around and slightly above approximately 60 $\mathrm{bpm}$. Assuming the heart rate is $60 \mathrm{bpm}$ and the pullback speed is $0.5 \mathrm{~mm} / \mathrm{s}$, Intelligate will then select enddiastolic ICUS images at $0.5 \mathrm{~mm}$ intervals. The longitudinal resolution of Intelligate is thus approximately half that of hardware ECG-gated studies. This did not result in any measurable difference of analysis results.

Since Intelligate can be applied retrospectively offline, it will be possible to re-analyze ICUS studies from previous trials that have been performed with non-gated datasets.

\section{Conclusion}

Fully automatic retrospective image-based ICUS enddiastolic gating is feasible, resulting in similar quantitative results as hardware based ECG-gated ICUS studies.

\section{References}

[1] Fitzgerald PJ, St Goar FG, Connolly AJ, Pinto FJ, Billingham ME, Popp RL, et al. Intravascular ultrasound imaging of coronary arteries. Is three layers the norm? Circulation 1992;86(1):154-8.

[2] Bruining N, von Birgelen C, de Feyter PJ, Ligthart J, Li W, Serruys PW, et al. ECG-gated versus nongated threedimensional intracoronary ultrasound analysis: implications for volumetric measurements. Cathet Cardiovasc Diagn 1998;43(3):254-60.

[3] Hamers R, Bruining N, Knook M, Sabate M, Roelandt JRTC. A Novel Approach to Quantitative Analysis of Intra Vascular Ultrasound Images. In: Murray A, editor. Computers in Cardiology; 2001 Sep 23-26; Rotterdam, The Netherlands: IEEE; 2001. p. 589-592.

Address for correspondence.

SA de Winter

Erasmus Medical Centre/Room H553

Dr. Molewaterplein 40

3015 GD Rotterdam

The Netherlands

E-mail: s.dewinter@erasmusmc.nl 